\title{
A New Approach on Vibrating Horns Design
}

\author{
Maria Violeta Guiman and Ioan Călin Roşca \\ Department of Mechanical Engineering, Faculty of Mechanical Engineering, Transilvania University of Brașov, 29 Eroilor Av., \\ 500036 Brașov, Romania \\ Correspondence should be addressed to Ioan Călin Roşca; icrosca@unitbv.ro
}

Received 5 February 2017; Revised 1 June 2017; Accepted 19 June 2017; Published 7 August 2017

Academic Editor: Tai Thai

Copyright ( $) 2017$ Maria Violeta Guiman and Ioan Călin Roşca. This is an open access article distributed under the Creative Commons Attribution License, which permits unrestricted use, distribution, and reproduction in any medium, provided the original work is properly cited.

\begin{abstract}
An optimization method of the vibrating horns is presented considering the smallest action principle and the attached cutting tool mass. The model is based on Webster's wave propagation equation and as an objective function the minimization of the volume in structural equilibrium conditions was considered. The considered input parameters were working frequency, maximum crosssectional area, magnification coefficient, and the attached mass. At the end of the study, a new shape function of the horn's cross section is obtained. The particularity of the new obtained shape is given by the nodal point position that is the same with the position of the maximum cross-sectional area. The obtained horn was analyzed from the modal point of view using theoretical and experimental methods. As theoretical methods, both the state-space method and the finite element method were used. An experimental setup for frequency response function determination was developed using a random input signal. The verification of the magnitude value was done considering a harmonic steady-state signal. The recorded values were compared with the predicted values. The numerical simulations and tests support the validity of the assumptions used in the horns optimization design.
\end{abstract}

\section{Introduction}

Ultrasonic horns are axisymmetric devices that provide, with high efficiency, acoustic energy developed by a transducer that is working based on the magnetostriction effect, to a tool. These devices can be themselves tools or can be used with a tool attached at the small end, and thus they are tied with high-power applications of ultrasound, like cutting [1$3]$, drilling [4-6], turning [7], welding [8, 9], and so forth, or for machining different materials as carbon fiber reinforced composites [10-12].

From a physical point of view, the work of such a system consists of an initial signal, emitted by a transducer at a given frequency, with an amplitude $u_{0}$, which is mechanically amplified by the horn, with an imposed magnitude coefficient q. The whole system is working in a resonance regime at an imposed frequency. The mechanical magnification of the input signal amplitude is generated by the horn varying cross section from an initial value $S_{0}$, in the origin of the reference system, to an end one value $S_{L}$ (Figure 1).

Generally, the cross section variation is given by different mathematical functions (exponential [13], linear [14], tapered (or stepped) [15], catenoidal [14], Bézier [16], Gaussian, etc.). Studies concerning the wave propagation and basic concepts of horns design are presented in different papers [17-21].

A problem that is associated with the horns design refers to the shape optimization. The optimization procedure is connected with design parameters like frequency working values [21], amplitude of the signal [22, 23], load transferred in the manufacturing area [24], the objective function [25, 26], the impact of different design variables on the objective function [27], combined signals transmitted in the horn [28], and so forth.

In the present paper, we present an approach based on the principle of the least action that takes into consideration as an objective function the volume minimization. The considered input parameters are the working frequency, the maximum cross-sectional area, the magnification coefficient, and the attached mass.

\section{Mathematical Model}

In case of ultrasonic horns, a planar stress waves state is assumed. The general equation that describes one-dimensional 


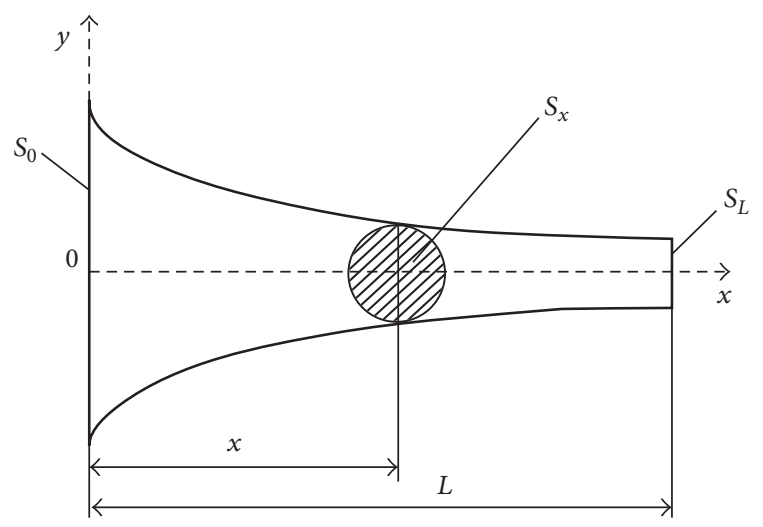

FIGURE 1: The solid horn geometry.

propagation, in the longitudinal direction, of ultrasonic waves along a rigid horn with a variable cross-sectional area is Webster's horn equation [29]:

$$
\frac{\partial^{2} u(x, t)}{\partial x^{2}}+\frac{\partial u(x, t)}{\partial x} \frac{\partial}{\partial x}\left(\ln S_{x}\right)=\frac{1}{c^{2}} \frac{\partial^{2} u(x, t)}{\partial t^{2}},
$$

where $u(x, t)$ is the signal, $S_{x}=S(x)$ represents the crosssectional area of the considered horn at the distance $x$ from the reference (point 0 ), and $c$ is the wave velocity in the horn's material.

One of the widely used techniques to find the explicit analytical solutions of the linear partial differential equations is the method of variables separation [30]. The method assumes that the solution of (1) can be written as a product of two functions, one in space domain $u(x)=u_{x}$ and the other one in time domain $u(t)=u_{t}$ :

$$
u(x, t)=u(x) u(t)=u_{x} u_{t}
$$

Introducing solution (2) in Webster's equation (1), the new shape is obtained:

$$
\frac{u_{x}^{\prime \prime}}{u_{x}}+\frac{d}{d x}\left(S_{x}\right) \frac{u_{x}^{\prime}}{u_{x}}=\frac{1}{c^{2}} \frac{\ddot{u}_{t}}{u_{t}},
$$

where the following notations were done: $u_{x}^{\prime \prime}=d^{2} u_{x} / d x^{2}$, $u_{x}^{\prime}=d u_{x} / d x$, and $\ddot{u}_{t}=d^{2} u_{t} / d t^{2}$.

This way, in (3), there were separate variables on each side of the equation.

The congruency of both sides, left and right, of (3) is achieved only if the two functions are equal with the same separation constant $\beta$. It is considered as a constant value $\beta=-\omega^{2}$, where $\omega$ is the angular wave frequency $(\mathrm{rad} / \mathrm{s})$.

Thus, from (3), we obtained the following ordinary differential equations:

$$
\begin{aligned}
\ddot{u}_{t}+\omega^{2} u_{t} & =0 ; \\
u_{x}^{\prime \prime}+\frac{d}{d x}\left(S_{x}\right) u_{x}^{\prime}+k^{2} u_{x} & =0,
\end{aligned}
$$

The general solution of the time domain equation from (4) is given by

$$
u_{t}=D_{1} \cos \omega t+D_{2} \sin \omega t
$$

with the initial conditions

$$
\begin{aligned}
\left.u_{t}\right|_{t=0} & =u_{0} \\
\left.\frac{d u_{t}}{d t}\right|_{t=0} & =0
\end{aligned}
$$

where $u_{0}$ is the initial amplitude of the ultrasonic wave.

Thus, solution (5) becomes

$$
u_{t}=u_{0} \cos \omega t
$$

and the total solution (2) becomes

$$
u(x, t)=u(x) u(t)=u_{x} u_{t}=u_{x} u_{0} \cos \omega t
$$

In case of a steady-state mode, considering the assumption of an infinitesimal motion in a beam made of nondissipative medium and with a variable cross-sectional area, the second equation from (4), which represents the plane wave motion, can be rewritten as

$$
\frac{d}{d x}\left(S_{x} \frac{d u_{x}}{d x}\right)+k^{2} S_{x} u_{x}=0
$$

It is considered that the maximum diameter of the horn should not exceed one-quarter of the wavelength $\lambda$ and the maximum cross section is denoted as $S_{m}$. Thus, we can write the condition that, on the whole length of the horn, the cross section value has to be smaller than the maximum cross section:

$$
S(x) \leq S_{m}
$$

Considering relation (10), one can write the following mathematical relation:

$$
S_{m}-S(x)-a^{2}(x)=0,
$$

where $a(x)$ is an unspecified function of the variable $x$. In the following, the notations $a(x)=a_{x}$ and $S(x)=S_{x}$ will be used.

An optimization problem of the horn's shape refers to minimizing the horn's volume for a given initial set of design data. Based on the principle of the least action, one can write the action relation as

$$
A=\int_{t_{1}}^{t_{2}}(T-V) d t
$$

where $T$ is the kinetic energy and $V$ represents the potential energy.

Considering the wave shape as

$$
u=u(x, t)=u(x) \cos (\omega t)=u_{x} \cos (\omega t)
$$


for a beam of length $L$ with attached mass at one end, the case of an ultrasonic horn with the attached tool, the total energies of the system horn tool, mentioned in (12), are given by

$$
\begin{aligned}
& T=\frac{1}{2} \int_{0}^{L}\left(m_{x} \dot{u}^{2}\right) d x+\frac{M_{t} \dot{u}^{2}(L)}{2}, \\
& V=\frac{1}{2} \int_{0}^{L}\left(\frac{E_{h} m_{x}}{\rho_{h}}\right)\left(u^{\prime}\right)^{2} d x+\frac{\sigma^{2}}{2 E_{t}} V_{t},
\end{aligned}
$$

where $m_{x}$ is the specific mass of the horn, $M_{t}$ is the attached mass (toll mass), $E_{h}$ is Young's modulus of the horn material, $E_{t}$ is Young's modulus of the tool material, $V_{t}$ is the volume of the attached tool, $\rho_{h}$ represents the horn material density, $\dot{u}=d u / d t$, and $u^{\prime}=d u / d x$.

Considering a period, action (12) becomes

$$
\left.A\right|_{t_{1}} ^{t_{2}+2 \pi / \omega}=\bar{A}=\pi(\bar{T}-\bar{V})
$$

with

$$
\begin{aligned}
& \bar{T}=\frac{\omega^{2}}{2}\left[\int_{0}^{L} \rho \cdot S \cdot u^{2} d x+M_{t} u^{2}(L)\right], \\
& \bar{V}=\frac{1}{2}\left[\int_{0}^{L} E_{h} \cdot\left(u^{\prime}\right)^{2} \cdot S d x+\sigma \cdot u^{\prime}(L) \cdot V_{t}\right] .
\end{aligned}
$$

Based on [31, 32], the following functional is defined:

$$
\mathbf{I}=\bar{T}-\bar{V}+\alpha_{1} V+\int_{0}^{L} \alpha_{x}\left(S_{m}-S_{x}-a_{x}^{2}\right) d x,
$$

where $\alpha_{1}$ and $\alpha_{x}=\alpha(x)$ are Lagrange multipliers and $V$ is the volume of the horn, given by

$$
V=\int_{0}^{L} S_{x} d x
$$

The optimization problem has a solution for the imposed limit values if the functional I given by (17) has to be stationary related to the variations of $u_{x}$ and to the design parameter $S_{x}$. Thus, based on (18), it is necessary for Euler's equations [33] to be satisfied:

$$
\begin{gathered}
\frac{\partial \mathbf{I}}{\partial u_{x}}-\frac{d}{d x} \frac{\partial \mathbf{I}}{\partial u_{x}^{\prime}}=0, \\
\frac{\partial \mathbf{I}}{\partial S_{x}}-\frac{d}{d x} \frac{\partial \mathbf{I}}{\partial S_{x}^{\prime}}=0, \\
\frac{\partial \mathbf{I}}{\partial a_{x}}-\frac{d}{d x} \frac{\partial \mathbf{I}}{\partial a_{x}^{\prime}}=0,
\end{gathered}
$$

where $u_{x}^{\prime}=d u_{x} / d x, S_{x}^{\prime}=d S_{x} / d x$, and $a_{x}^{\prime}=d a_{x} / d x$.

Considering the functional given by (17) and (19), one can obtain the following differential equations:

(a) From the first equation in (19) results

$$
\begin{gathered}
\frac{\omega^{2}}{2} 2 u_{x} \rho S_{x}-\frac{d}{d x}\left[-\frac{E_{h}}{2} 2 u_{x}^{\prime} S_{x}\right] \\
=\omega^{2} u_{x} \rho S_{x}+E_{h}\left(S_{x} u_{x}\right)^{\prime}=0
\end{gathered}
$$

which when divided by $E_{h}$ becomes

$$
\left(S_{x} u_{x}^{\prime}\right)^{\prime}+\frac{\rho_{h} \omega^{2}}{E_{h}} S_{x} u_{x}=0
$$

Considering the velocity of the longitudinal wave in the horn as $c_{l}=\sqrt{E_{h} / \rho_{h}}$ and the wave number $k=\omega / \mathcal{c}_{l},(21)$ can be rewritten as

$$
\left(S_{x} u_{x}^{\prime}\right)^{\prime}+k^{2} S_{x} u_{x}=0
$$

(b) From the second equation, one obtains

$$
\frac{\omega}{2} \rho_{h} u_{x}^{2}-E_{h}\left(u_{x}^{\prime}\right)^{2}+2 \alpha_{1}-2 \alpha_{x}=0
$$

which when divided by $E_{h}$ becomes

$$
k u_{x}^{2}-\left(u_{x}^{\prime}\right)^{2}+\frac{2}{E_{h}} \alpha_{1}-\frac{2}{E_{h}} \alpha_{x}=0 .
$$

(c) From the third equation results, it is found that

$$
2 \alpha_{x} a_{x}=0
$$

Analyzing the previous equations, one can obtain the following remarks about the optimal design of the ultrasonic horn:

(a) Equation (21) is identical to the longitudinal wave propagation equation along a horn and it is available along the whole horn.

(b) Equation (25) leads to two other conditions:

$$
\begin{aligned}
\alpha_{x} & =0 \\
\text { or } a_{x} & =0 .
\end{aligned}
$$

Conditions (26) involve the following aspect: the function $a_{x}$ is different from zero on the whole length of the horn except for one point, where $S_{x}=S_{m}$.

Consequently, for (25) to be satisfied for any point in the range $0 \leq x \leq L$, it is necessary to have the first condition from (26) $\alpha_{x}=0$ in the abovementioned range. Moreover, all Lagrange's coefficients have to be constants.

(a) From (24) and (26), the following is obtained:

$$
\alpha_{x}=\frac{E_{h}}{2}\left[k^{2} u_{x}^{2}-\left(u_{x}^{\prime}\right)^{2}+\frac{2 \alpha_{1}}{E_{h}}\right]=0
$$

or

$$
\left(u_{x}^{\prime}\right)^{2}-k^{2} u_{x}^{2}-\frac{2 \alpha_{1}}{E_{h}}=0
$$

\section{The Solution of the Differential Equation}

The solution of (28) can be found considering the initial functional conditions of the ultrasonic horn. The following are considered as initial conditions: 
(a) The length of the horn has to be equal to a wavelength.

(b) To achieve the assumption that the propagated waves are only longitudinal ones, a maximum value is considered for the magnification coefficient $q=5$ [17].

(c) The initial value of the signal amplitude, at the connection point between the transducer and the horn $(x=0)$, is $u_{0}$ equal to the emitted signal by the transducer:

$$
u(x)=u(0)=u_{0} .
$$

(d) Considering the initial signal and the magnification coefficient, the signal at the end of the horn (for $x=L$ ) is given by

$$
u(x)=u(L)=-q u_{0} .
$$

(e) In (30), the negative value (-) is considered in order to obtain a nodal point in a position along the horn length.

(f) Between the two ends of the horn, there is no other extreme of the signal except for the maximum developed at the end of the horn, in the connection point with the tool.

(g) The following condition is considered [32]:

$$
m u_{x=L}^{\prime}-k^{2} M u_{x=L}=0,
$$

where $m$ is the mass on the unit length, at the end of the horn, $u_{x=L}^{\prime}$ is the strain at the end of the horn, $M$ is the attached mass, and $u_{x=L}$ is the signal at the end of the horn, given by (30).

Denoting

$$
C=\frac{2}{E} \alpha_{1}
$$

equation (28) can be rewritten as

$$
\left(u^{\prime}\right)^{2}-k^{2} u^{2}-C=0
$$

and integrating it with the derivative method, one can obtain the following equation [34]:

$$
2 u^{\prime} u^{\prime \prime}-k^{2} 2 u u^{\prime}=0 .
$$

Dividing (34) by $u^{\prime} \neq 0$ results in the differential equation

$$
u^{\prime \prime}-k^{2} u=0
$$

with the solution

$$
u(x)=C_{1} e^{k x}+C_{2} e^{-k x} .
$$

Introducing solution (36) in (33), after some calculations, the following is obtained:

$$
\begin{aligned}
-4 k^{2} C_{1} C_{2} & =C, \\
C_{1} & =-\frac{C}{4 k^{2} C_{2}} .
\end{aligned}
$$

Considering (38) in solution (36) results in

$$
u(x)=-\frac{C}{4 k^{2} C_{2}} e^{k x}+C_{2} e^{-k x} .
$$

The constants $C$ and $C_{2}$ can be found considering the initial conditions mentioned above, for $x=0$. Combining (38) and (30), the following relation is obtained:

$$
u_{0}=-\frac{C}{4 k^{2} C_{2}}+C_{2},
$$

which leads to the following relation for the two values of the constant $C_{2}$ as a function of $C$ :

$$
C_{2}^{1,2}=\frac{k u \pm \sqrt{k^{2} u_{0}^{2}+C}}{2 k} .
$$

Considering (41) and (40), for both values $C_{2}^{1,2}$, the same dependence is obtained between the constants $C$ and $C_{2}$ :

$$
C=4 C_{2} k^{2}\left(C_{2}-u_{0}\right)
$$

Introducing the constant $C$, given by (42), in solution (39) leads to the following relation of the amplitude $u(x)$ :

$$
u(x)=u_{0} e^{k x}-C_{2}\left(e^{k x}-e^{-k x}\right) .
$$

The constant $C_{2}$ can be found considering conditions (39) and (40):

$$
\rho_{h} S_{h L}\left[u_{0} e^{k L}-C_{2}\left(e^{k L}+e^{-k L}\right)\right]+k L_{t} \rho_{t} S_{t} q u_{0}=0,
$$

where $\rho_{h}$ is the density of the horn's material, $S_{h L}$ is the cross section at the end of the horn, $S_{t}$ is the cross section of the tool $\left(S_{L}=S_{t}\right), L_{t}$ is the tool's length, $\rho_{t}$ is the density of the tool's material, and $q$ is the gain of the initial signal $u_{0}$.

From (44) results

$$
C_{2}=\frac{u_{0}\left(\rho_{h} S_{h L} e^{k L}+q k L_{t} S_{t} \rho_{t}\right)}{2 \rho_{h} S_{h L} \cosh (k L)} .
$$

Considering dependence (42), one can define the function of the space dependent amplitude component of the signal along the horn:

$$
\begin{aligned}
& u(x) \\
& \quad=u_{0}\left[e^{k x}-\frac{\rho_{h} S_{h L} e^{k L}+q k L_{t} S_{t} \rho_{t}}{\rho_{h} S_{h L}\left(e^{k L}+e^{-k L}\right)}\left(e^{k x}-e^{-k x}\right)\right] .
\end{aligned}
$$

In the following, we use the notation

$$
B=\frac{\rho_{h} S_{h L} e^{k L}+q k L_{t} S_{t} \rho_{t}}{\rho_{h} S_{h L}\left(e^{k L}+e^{-k L}\right)},
$$

and the signal function (46) becomes

$$
u(x)=u_{0}\left[(1-B) e^{k x}+B e^{-k x}\right] .
$$


As it is known, an important aspect in horns design is to find the position of the neutral point $x_{n}$. The neutral point defines the position of the cross section where the signal is zero. Thus, the neutral point can be easily found considering relation (48). Equalizing function (48) with zero, it is obtained that

$$
x_{\text {nod }}=\frac{1}{2 k} \ln \left(\frac{B}{B-1}\right) .
$$

\section{Cross Section Shape Definition}

From relation (48), we obtained the first two derivatives of the space component of the signal:

$$
\begin{aligned}
u^{\prime}(x) & =k u_{0}\left[(1-B) e^{k x}-B e^{-k x}\right], \\
u^{\prime \prime}(x) & =k^{2} u_{0}\left[(1-B) e^{k x}+B e^{-k x}\right] .
\end{aligned}
$$

Equation (9) can be rewritten as

$$
\frac{S^{\prime}}{S}=-\frac{u^{\prime \prime}+k^{2} \cdot u}{u^{\prime}}
$$

Considering relations (50) in (51) results in the new formula

$$
\frac{S_{x}^{\prime}}{S_{x}}=-\frac{2 u_{0} k^{2}\left[(1-B) e^{k x}+B e^{-k x}\right]}{u_{0} k\left[(1-B) e^{k x}-B e^{-k x}\right]} .
$$

Defining the function $g(x)=g_{x}$ as

$$
g(x)=(1-B) e^{k x}-B e^{-k x},
$$

(52) becomes

$$
\frac{S_{x}^{\prime}}{S_{x}}=-2 \cdot \frac{g_{x}^{\prime}}{g_{x}} .
$$

Integrating (54) results in a constant, denoted by $D$ :

$$
\ln \left(S_{x}\right)=-2 \ln \left(g_{x}\right)+D,
$$

which depends on the initial geometrical conditions.

At the end of the horn, for $x=L$, the cross section becomes $S_{x}=S_{L}$ and it is obtained that

$$
D=\ln \left(S_{x}\right)+2 \ln \left(g_{L}\right),
$$

where $g_{L}$ is the value of the function $g(x)=g_{x}$, defined by (53), for $x=L$. in

Introducing the value of $D$ obtained by (56) in (55) results

$$
\ln \left(S_{x}\right)=-2 \ln \left(g_{x}\right)+\ln \left(S_{L}\right)+2 \ln \left(g_{L}\right),
$$

or

$$
\ln \left(\frac{S_{x}}{S_{L}}\right)=-2 \ln \left(\frac{g_{x}}{g_{L}}\right),
$$

and the cross section at the distance $x$ can be calculated as

$$
S_{x}=S_{L}\left(\frac{g_{L}}{g_{x}}\right)^{2} .
$$

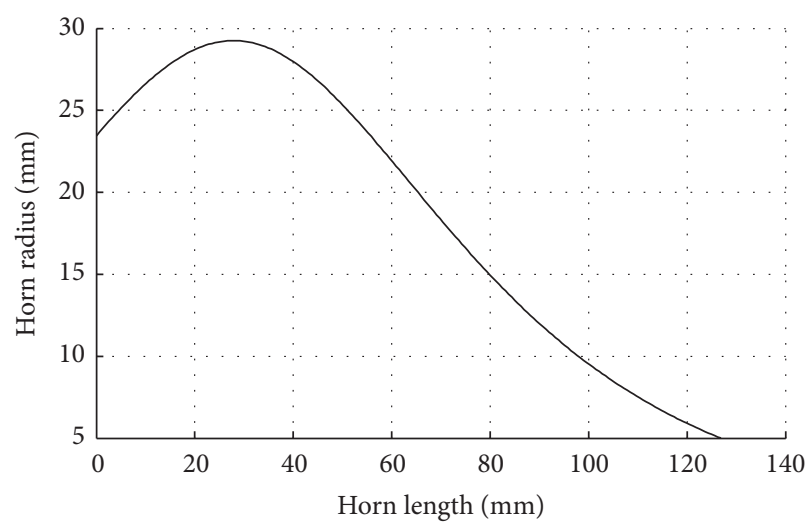

FIgURE 2: The half-horn shapes along the longitudinal axis.

An interesting design aspect is represented by the value and position of the maximum cross section. Thus, we considered the condition that the first derivative of function (59) is equal to zero. This condition leads to the relation

$$
g_{x}^{\prime}=0
$$

with similar solution to (49).

It can be concluded that the position of the maximum cross section is in the same place as the nodal point.

The end cross section can be found as

$$
S_{L}=S_{m}\left[\frac{g_{n}}{g_{L}}\right]^{2}
$$

and introducing (61) in (59) results in the relation of the cross section value in a point situated at the distance $x$ from the system origin, as a function of the maximum cross section:

$$
S_{x}=S_{m}\left(\frac{g_{n}}{g_{x}}\right)^{2} .
$$

Taking into consideration relation (62), a script in Matlab was made to generate the shape of the horn made of steel. The input parameters were the resonance frequency $f_{r}=$ $19900 \mathrm{~Hz}$, the wave velocity in steel $c=50550 \mathrm{~m} / \mathrm{s}$, the horn's material density $\rho_{h}=7850 \mathrm{~kg} / \mathrm{m}^{3}$, the end radius of the horn (connection surface with the tool) $r_{h L}=0.005 \mathrm{~m}$, the magnification coefficient $q=5$, and tool radius equal to the end horn radius $\left(r_{t}=r_{h L}\right)$. The tool was considered to be made of steel.

Based on the script written in Matlab, the shape presented in Figure 2 was obtained, and in Figure 3 the physical model of the horn is presented.

Based on the same script, the following design data were found: the radius in the origin of the system $(x=0) r_{0}=$ $29.256 \mathrm{~mm}$, the largest radius $r_{m}=31.721 \mathrm{~mm}$, and the position of the nodal point $x_{\text {nod }}=27.734 \mathrm{~mm}$.

Considering relation (48), using a script written in Matlab, we obtained the magnitude variation of the spatial components $u(x)$ of the considered signal (13) (Figure 4).

As it can be seen from Figure 4, the nodal point is at the coordinate found by relation (49). 


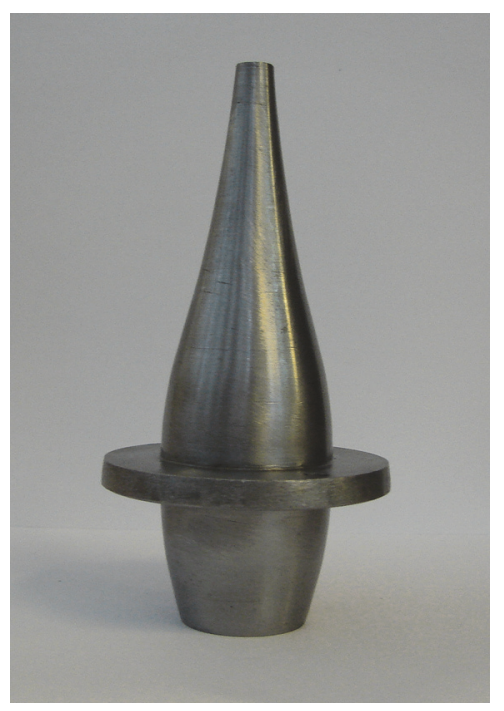

FIGURE 3: The physical model of the horn.

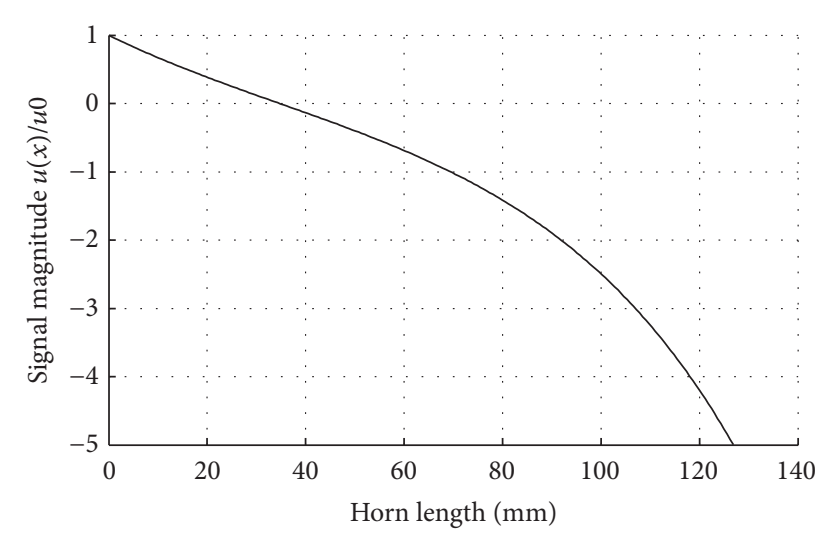

FIGURE 4: The signal magnitude along the horn.

\section{Theoretical Modal Analysis}

5.1. State-Space Approach of the Optimized Model. The modal behavior can be described in many ways. Two of them are the state-space approach and transfer function representation. The differences between these two methods were described in $[35,36]$. In the present paper, we considered as a theoretical modal analysis the state-space approach, and for the experimental modal analysis the FRF representation was made.

The state-space method is often used in dynamic system analysis. This method can be applied in the case of lumped systems and it is based on the concept of state vector that consists of two components: the vector of displacement and the vector of the velocities. Practically, the " $n$ " second-order coupled equations that describe the dynamic behavior of a lumped system are transformed in a set of $2 n$ first-order coupled equations [37-39].

The ultrasonic horn can be considered as a linear timeinvariant (LTI) system that can be approximate with a lumped mass system. Thus, the obtained horn (Figure 3 ) is divided into " $n$ " elements with the same length $l_{i}$, where $l_{i}=L / n$,

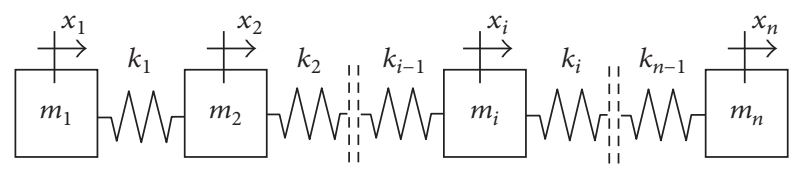

Figure 5: The lumped model of the horn.

with $L$ being the total length of the horn equal to a quarter of the wavelength. Considering the case of the free-free system, as is in the experimental modal analysis, the horn can be modeled as a lumped system made of " $n$ " masses connected with springs (Figure 5).

We considered 200 masses with a length of $l_{i}=6.344$. $10^{-4} \mathrm{~m}$ in each part. The obtained components are, taking into consideration the shape (Figure 3), small frusta of cone elements.

The components of the modal model are established considering the following assumptions:

(a) The stiffness of the springs was considered to be equal to the equivalent stiffness of each element based on the relation

$$
k_{i}=\frac{E S_{a i}}{l_{i}},
$$

where $S_{a i}$ represents the average area of the region of length $l_{i}$ :

$$
S_{a i}=\frac{S_{i}+S_{i+1}}{2}
$$

with both $S_{i}$ and $S_{i+1}$ calculated using relation (62).

(b) The mass $m_{i}$ of each small frustum of cone element was found considering the classical relation of the density using for volume the areas $S_{i}$ and $S_{i+1}$ and the length $l_{i}$.

Considering the d'Alembert principle, the motion equations for the system from Figure 5 are given by

$$
\begin{gathered}
m_{1} \ddot{x}_{1}+k_{1} x_{1}-k_{1} x_{2}=0, \\
m_{2} \ddot{x}_{2}+\left(k_{1}+k_{2}\right) x_{2}-k_{1} x_{1}-k_{2} x_{3}=0, \\
m_{3} \ddot{x}_{3}+\left(k_{2}+k_{3}\right) x_{3}-k_{2} x_{2}-k_{3} x_{4}=0, \\
\vdots \\
m_{i} \ddot{x}_{i}+\left(k_{i-1}+k_{i}\right) x_{i}-k_{i-1} x_{i-1}-k_{i} x_{i+1}=0, \\
\vdots \\
m_{n} \ddot{x}_{n}+k_{n-1} x_{n}-k_{n-1} x_{n-1}=0 .
\end{gathered}
$$


System (65) can be rewritten in matrix form as follows:

$$
\begin{aligned}
& {\left[\begin{array}{ccccccc}
m_{1} & 0 & 0 & \cdots & \cdots & \cdots & 0 \\
0 & m_{2} & 0 & \cdots & \cdots & \cdots & 0 \\
0 & 0 & m_{3} & \cdots & \cdots & \cdots & 0 \\
\vdots & \vdots & \vdots & \vdots & \vdots & \vdots & \vdots \\
0 & 0 & 0 & \cdots & m_{i} & \cdots & 0 \\
\vdots & \vdots & \vdots & \vdots & \vdots & \vdots & 0 \\
0 & 0 & 0 & \cdots & \cdots & \cdots & m_{n}
\end{array}\right]\left\{\begin{array}{c}
\ddot{x}_{1} \\
\ddot{x}_{2} \\
\ddot{x}_{3} \\
\vdots \\
\ddot{x}_{i} \\
\vdots \\
\ddot{x}_{n}
\end{array}\right\}} \\
& +\left[\begin{array}{ccccccc}
k_{1} & -k_{1} & 0 & \cdots & \cdots & \cdots & 0 \\
-k_{1} & k_{1}+k_{2} & -k_{2} & \cdots & \cdots & \cdots & 0 \\
0 & -k_{2} & k_{2}+k_{3} & -k_{3} & \cdots & \cdots & 0 \\
\vdots & \vdots & \vdots & \vdots & \vdots & \vdots & \vdots \\
\vdots & \vdots & \vdots & \cdots & \vdots & \cdots & 0 \\
\vdots & \vdots & \vdots & \vdots & \vdots & \vdots & 0 \\
0 & 0 & 0 & \cdots & \cdots & -k_{n-1} & k_{n-1}
\end{array}\right]\left\{\begin{array}{c}
x_{1} \\
x_{2} \\
x_{3} \\
\vdots \\
x_{i} \\
\vdots \\
x_{n}
\end{array}\right\}
\end{aligned}
$$$$
=\left\{\begin{array}{c}
0 \\
0 \\
0 \\
\vdots \\
0 \\
\vdots \\
0
\end{array}\right\}
$$

or

$$
\mathbf{M X}+\mathbf{K X}=\mathbf{0},
$$

from which it is obtained that

$$
\ddot{\mathbf{X}}=-\mathbf{M}^{-1} \mathbf{K X} \text {. }
$$

As the state vector, we considered the matrix vector

$$
\begin{aligned}
\Delta & =\left\{\delta_{1}, \delta_{2}, \ldots, \delta_{2 q}\right\}^{T}=\{\mathbf{X}, \dot{\mathbf{X}}\}^{T} \\
& =\left\{x_{1}, x_{2}, \ldots, x_{n}, \dot{x}_{1}, \dot{x}_{2}, \ldots, \dot{x}_{n}\right\}^{T},
\end{aligned}
$$

which, by derivation and considering (68), leads to the equation

$$
\dot{\Delta}=\left\{\begin{array}{l}
\dot{\mathbf{X}} \\
\ddot{\mathbf{X}}
\end{array}\right\}=\left[\begin{array}{cc}
\mathbf{0} & \mathbf{I} \\
-\mathbf{M}^{-1} \mathbf{K} & \mathbf{0}
\end{array}\right]\left\{\begin{array}{l}
\mathbf{X} \\
\dot{\mathbf{X}}
\end{array}\right\}=\mathbf{A} \Delta,
$$

where $\mathbf{I}$ is the unit matrix and $\mathbf{0}$ is the zeros matrix, both of $n \times n$ type.

The matrix $\mathbf{A}$ is defined as the state matrix or the transmission matrix and is a property of the system being

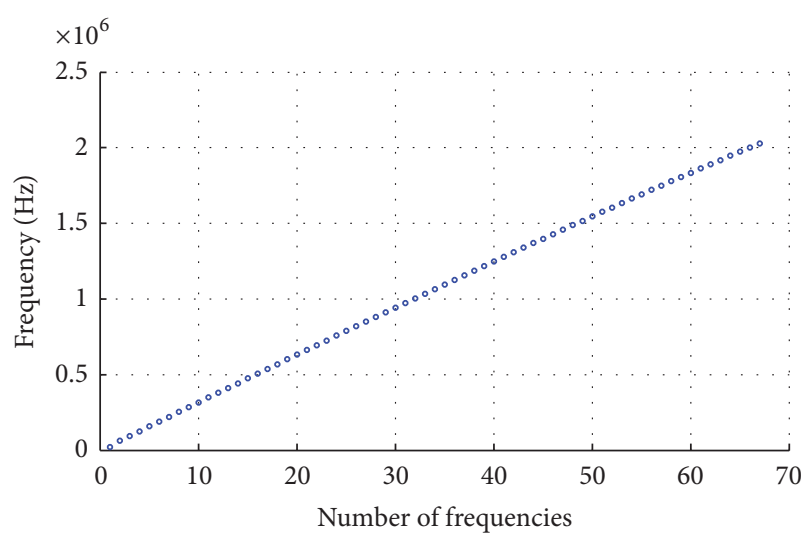

FIgURE 6: The natural frequencies obtained for the lumped model.

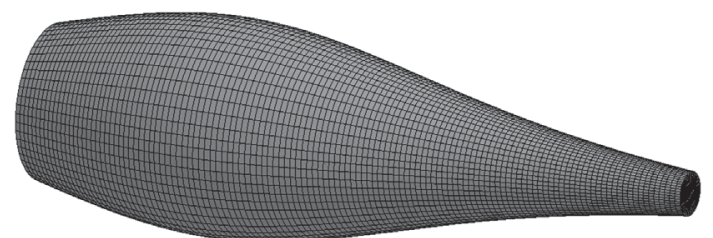

Figure 7: The finite element model of the horn.

defined by the structure of the system. The eigenvalues of the state matrix can be found based on the relation

$$
|\lambda \mathbf{I}-\mathbf{A}|=\mathbf{0}
$$

that is developed in an equation with unknowns $\lambda$.

If, in relation (71), the value $\lambda=j \omega_{n}$ is considered, where $j=\sqrt{-1}$ and $\omega_{n}$ is the natural frequency measured in $\mathrm{rad} / \mathrm{s}$, then the so-called characteristic equation is obtained, which gives the natural frequencies.

Thus, considering the equivalent system from Figure 5 with the associated motion equations (66), (67), and (70), one can find the natural frequencies of the system. The number and the precision of these frequencies depend on the number of the considered masses $m_{i}(i=\overline{1, n})$. Figure 6 presents the frequency values obtained for a model with 200 masses considering the method of eigenvalues of the transition matrix.

Figure 6 also presents some of the obtained natural frequencies. The first natural frequency found was $f_{n}=$ $19,837 \mathrm{~Hz}$, which is close to the designed frequency of $f_{r}=$ $19.9 \mathrm{kHz}$.

5.2. Finite Element Model. In the next step, the finite element model was considered. The used specialized software was ANSYS. The 3D model (Figure 7) was made based on (59).

For good convergence, we considered a mapped mesh consisting of first-order solid hexaelements with $1 \mathrm{~mm}$ length. We obtained 87,076 nodes and 75,254 elements. Mode extraction is carried out in the frequency range $18-24 \mathrm{kHz}$ using Block Lanczos option with double precision. Two pure longitudinal modes were obtained with the natural frequencies of $f=19,817 \mathrm{~Hz}$ (Figure 8) and $f=23,151 \mathrm{~Hz}$ (Figure 9). 
Freq. $=19817$

Contours of resultant displacement

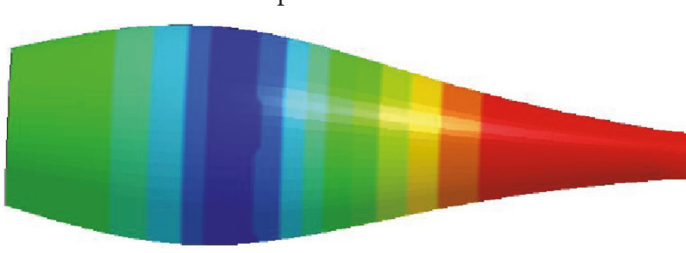

Resultant displacement $6.934 e-01$ $6.241 e-01$ $5.548 e-01$ $4.854 e-01$ $3.467 e-01$ $2.774 e-01$ $2.080 e-01$ $1.387 e-01$ $1.387 e-01$
$6.934 e-02$
$1.185 e-10$ $6.934 e-02$

Figure 8: The mode shape for the natural frequency of $f=19,817 \mathrm{~Hz}$.

Freq. $=23151$

Contours of resultant displacement

Resultant displacement $6.934 e-01$ $6.244 e-01$ $5.555 e-01$ $4.865 e-01$ $4.175 e-01-$ $3.485 e-01-$ $2.795 e-01-$ $2.105 e-01-$ $1.415 e-01$ $7.251 e-02$ $3.157 e-03$

FIGURE 9: The mode shape for the natural frequency of $f=23,151 \mathrm{~Hz}$.

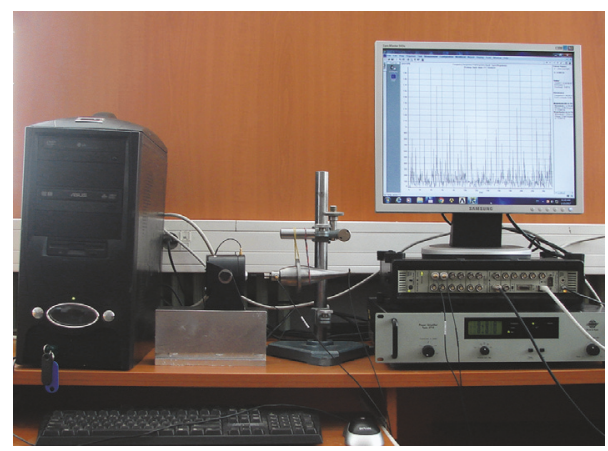

FIgURE 10: The modal testing setup.

\section{Experimental Modal Analysis}

The aim of the experimental modal analysis is to verify whether the considered imposed working frequency of $19.9 \mathrm{kHz}$ is one of the natural frequencies of the considered horn and whether the natural frequencies found by statespace approach are close to the real natural frequencies.

The method with random signal excitation given by an electrodynamic shaker (Figure 10) was considered [40]. The used equipment was a complete testing setup (Brüel \& Kjær) made of an integrated platform, PULSE 12, with a force transducer (type 8230) and an accelerometer (type 4397). The excitation signal was generated by the PULSE 12 platform and was amplified using the power amplifier type 2718 and then transmitted to the tested horn by the shaker type 4810 (Figure 11).

The random generated signal (the input signal) has a frequency span up to $25.6 \mathrm{kHz}$ with an excitation time of $500 \mathrm{~ms}$ (Figure 12). The Fourier spectrum of the input signal is shown in Figure 13.

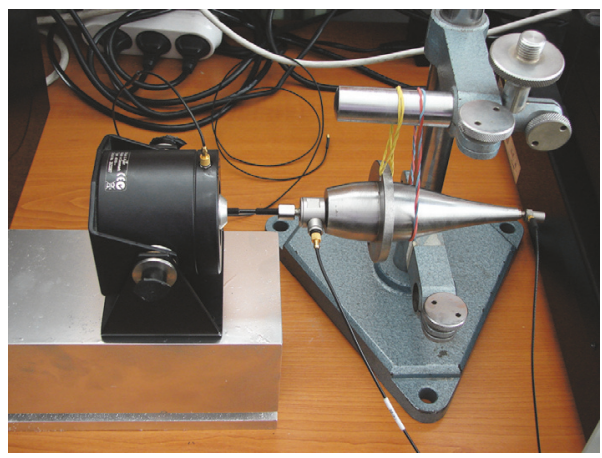

FIGURE 11: The detailed connection shaker horn.

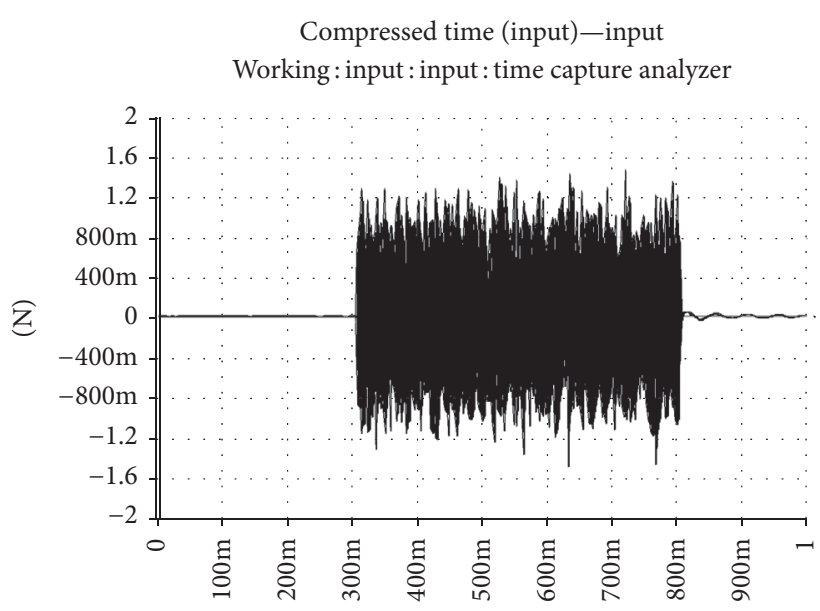

(s)

FIgURE 12: The input random signal in time domain. 


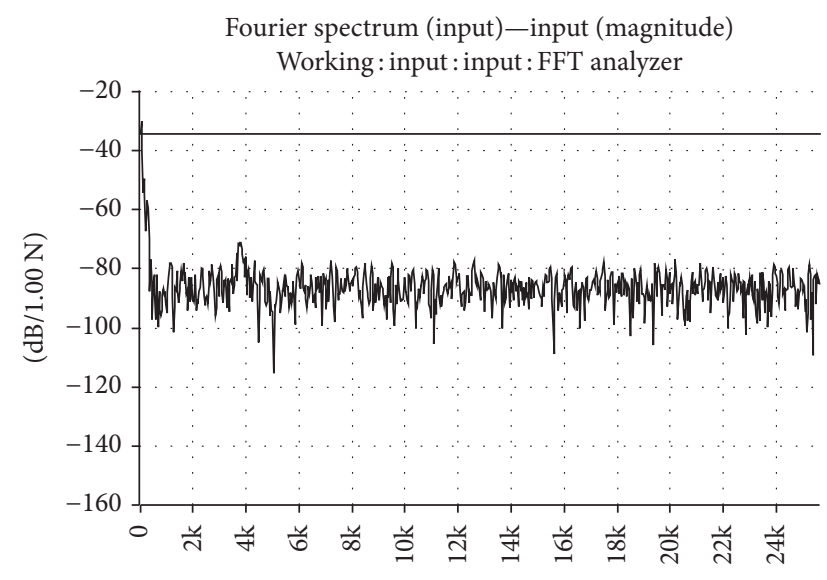

$(\mathrm{Hz})$

Figure 13: The Fourier spectrum of the input signal.

Frequency response $H_{1}$ (response, input)—input (magnitude) Working: input: input: FFT analyzer

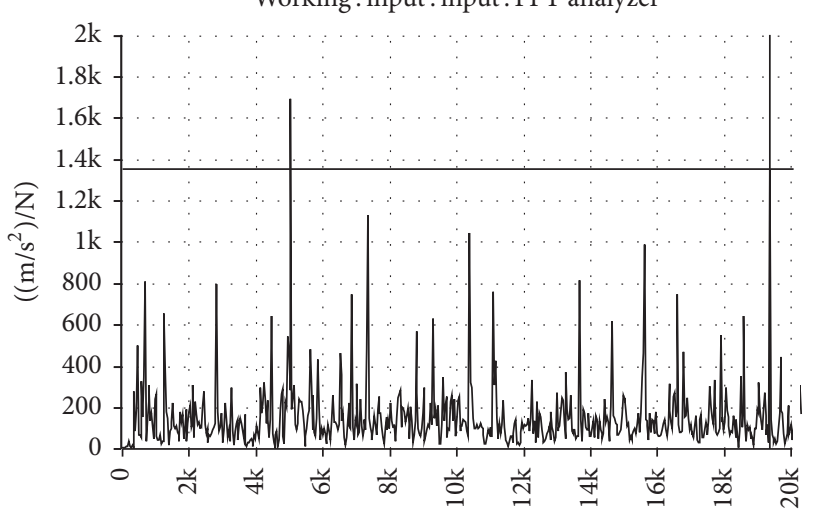

$(\mathrm{Hz})$

FIGURE 14: The frequency response function of the horn.

As it is known, the frequency response function estimation from the measured data represents the main step of the experimental modal analysis. The analysis is based on nonparametric estimators. In the present study, we considered the often used estimator $H_{1}$. The obtained frequency response function, for the estimator $H_{1}$, is presented in Figure 14.

The range of the measured frequencies was up to $20,000 \mathrm{~Hz}$ and the values are presented in Figure 15.

At the same time, based on the experimental modal analysis, we obtained the damping factors $\zeta$ for each natural frequency found. The distribution of these values is presented in Figure 16.

Considering the facilities of the Matlab software, a power function was defined, which can approximate the damping factor for different values of the frequency $f$ :

$$
\zeta(f)=321.9 f^{-0.7427}-0.07775 \text {. }
$$

Function (72) can be used to study the influence of the damping in case of horn vibration at different frequencies.

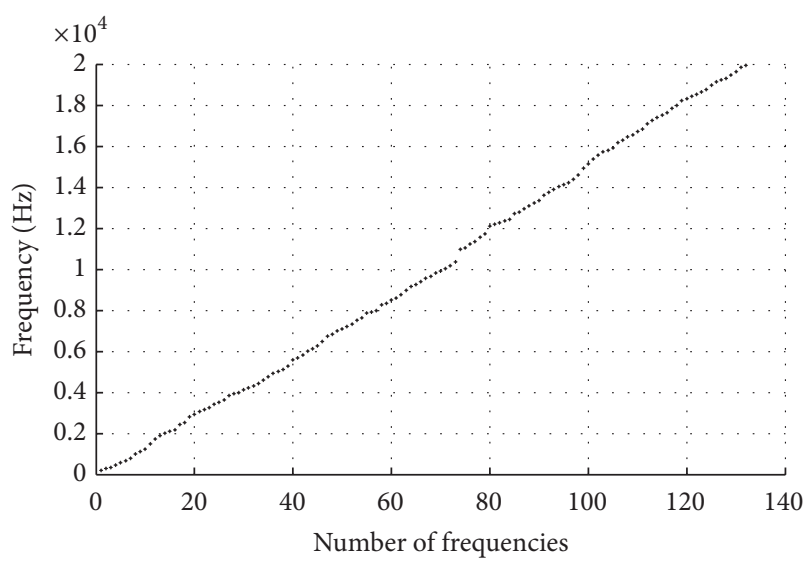

FIgURE 15: The frequencies obtained by experimental data.

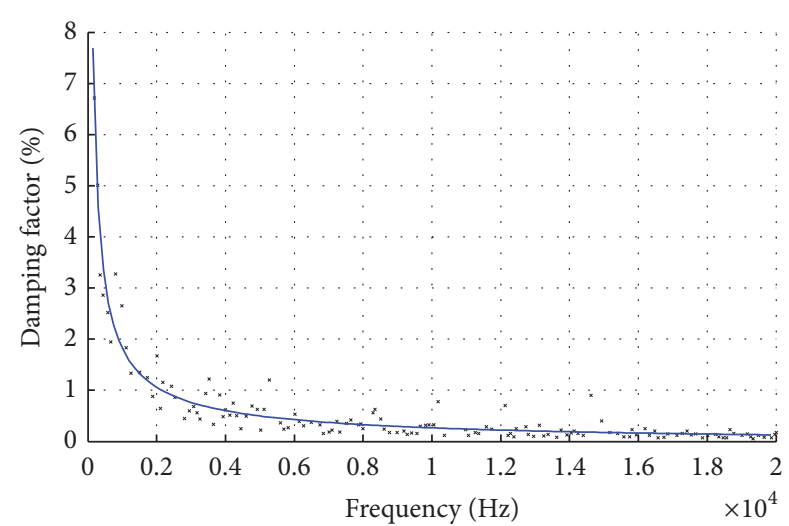

FIGURE 16: The damping factor values distribution versus natural frequencies.

\section{Testing the Designed Horn}

The test was designed to check whether the magnification of the designed horn is the initial considered $q=5$ for the resonance frequency of $19.9 \mathrm{kHz}$. The used setup is made of the same components as the setup used in the experimental modal analysis with the exception of the accelerometers. For testing, two Brüel \& Kjær accelerometers type 4517-002 were used. The signal was generated by a magnetostrictive transducer for a harmonic signal $y(t)=A \sin \omega t$ with a frequency $f=19.9 \mathrm{kHz}$. The input signal, represented in time domain, is shown in Figure 17, and the output signal (response) also in time domain is designed in Figure 18.

At the same time, for frequency control, we performed a check in frequency domain for both signals: the input (Figure 19) and the output (Figure 20).

Considering the values of the input and output signals (Figures 17 and 18), the magnification obtained by experiment is given by the following relation:

$$
q_{\text {real }}=\frac{u_{\text {output }}}{u_{\text {input }}}=\frac{42.78}{9.32}=4.59,
$$

which represents an error of about $8.2 \%$ between the considered value $q=5$ and the obtained value $q_{\text {real }}$. 


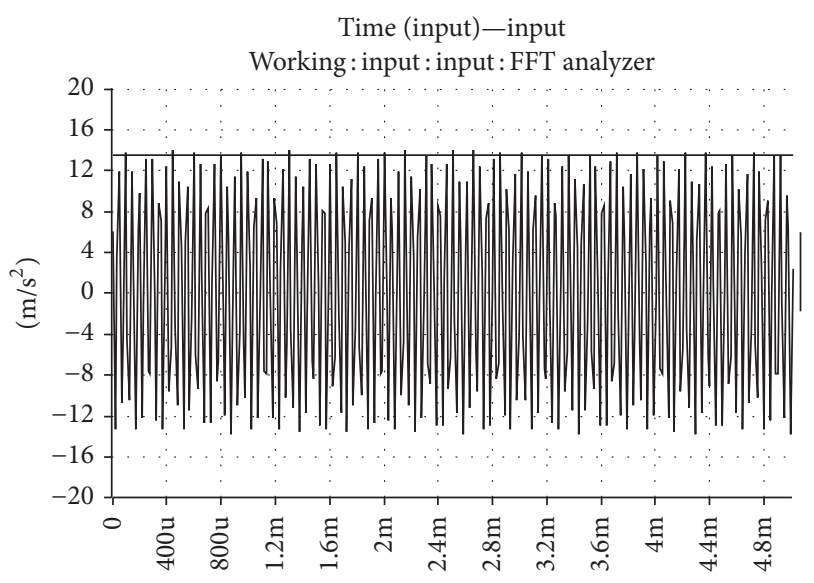

(s)

FIGURE 17: The input signal in time domain.

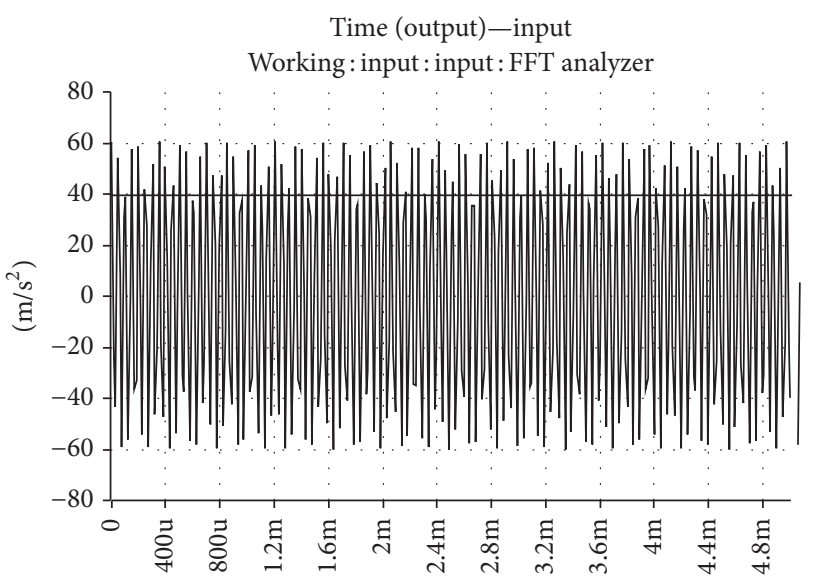

(s)

FIgURE 18: The output signal in time domain.

\section{Conclusions}

Based on the principle of the smallest action, an optimization of the shape of an ultrasonic horn was performed considering Webster's equation. The design was made considering a working resonance frequency of $f_{r}=19.9 \mathrm{kHz}$ and a magnification coefficient $q=5$, and the main assumption was that, through the horn and all attached coupling devices, only longitudinal plane waves propagate.

The considered boundary conditions were the classical ones referring to the amplitude of the signal, relations (29) and (30), to which a new one was added, relation (31), which highlights the influence of the tool mass, added at the end of the horn. This condition (31) is a particularity of the study.

The optimization procedure led to a new shape function (62), with the space dependent signal component function (48) and the position of the neutral point $x_{n}$ (49) being determined at the same time. Comparing the obtained shape with the classical shape, it can be seen that it is a different one, with the largest diameter in the neutral point. In the case of the other horns' shape, the largest diameter is at one end.

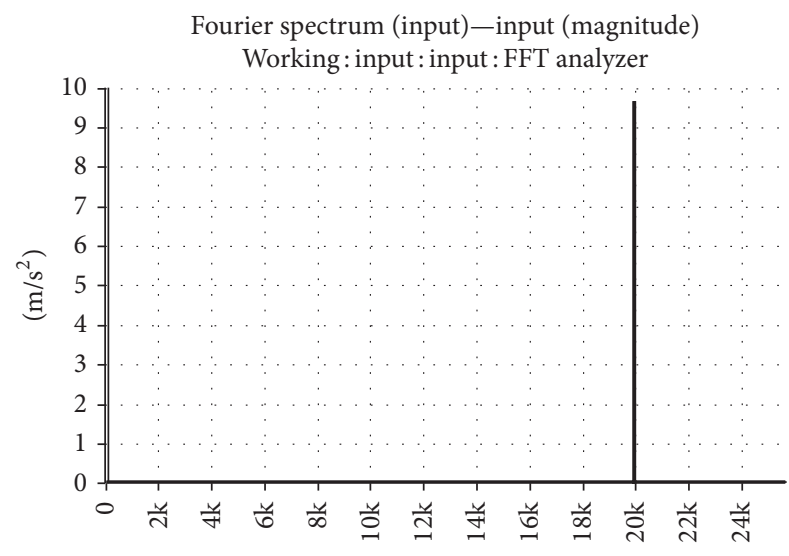

$(\mathrm{Hz})$

FIGURE 19: The input signal in frequency domain.

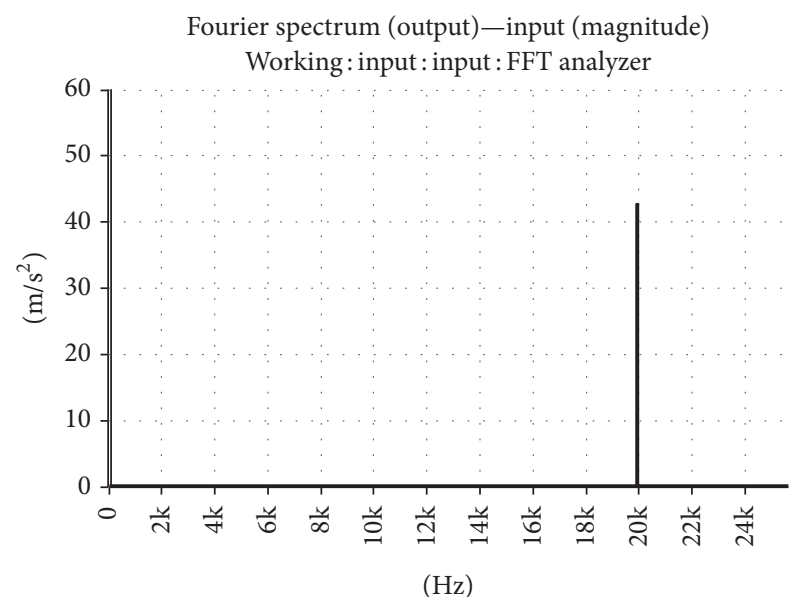

FIgURE 20: The output signal in frequency domain.

The natural frequencies were determined both in simulation (theoretically) and experimentally. In the case of theoretical modal analysis, the state-space representation was used. The horn was divided into 200 masses connected by springs (Figure 5) and the eigenvalues of the transmission matrix of system (71) were calculated. The first obtained value of the natural frequency was the imposed resonant frequency $f_{1, s s}=19,982 \mathrm{~Hz}$.

The second considered theoretical approach in theoretical modal analysis was the finite element method. A model with 75,254 elements was designed, and based on ANSYS software facilities, two natural frequencies were found around the designed resonance frequency (Figures 8 and 9), both for the longitudinal mode shape. In order to provide clarity to the results, a run was made in double precision mode.

The experimental modal analysis offered the possibility of finding a set of natural frequencies (Figure 16) and the corresponding values of the damping factor. Considering the facilities of the Matlab tool, a function of damping factor variation was obtained according to the frequencies values (72) useful in the dynamic analysis. As it can be seen, a 
natural frequency around the resonance designed frequency was found.

At the end, the horn was tested to check whether the magnification coefficient is the same as the designed one. A harmonic signal with the frequency value equal to the resonance frequency was considered as the input, and the output signal was measured. The found magnification coefficient is close to the designed one (72).

In the frame of the test, the attached tool was not considered. It is important for the assembly transducer-horntool to work in resonance regime and each part to work within the parameters for which it was designed.

The aim of the test was to check whether the considered horn, designed in the proposed set of boundary conditions (29), (30), and (31), generates the initial designed mechanical magnification of the input signal.

The influence of the attached tool mass is the particular design element that was taken into consideration compared with the other methods of optimization and design.

Considering the abovementioned aspects, it can be concluded that the numerical simulations and tests that were done support the validity of the assumptions used in the horn's optimization design.

The proposed model offers as an advantage a more convenient placement of the nodal point that is situated in the same place as the largest cross section.

The described design and optimization method offer the possibility of obtaining the shape based on the main working parameters.

\section{Conflicts of Interest}

The authors declare that they have no conflicts of interest.

\section{References}

[1] V. K. Astashev and V. I. Babitsky, "Ultrasonic cutting as a nonlinear (vibro-impact) process," Ultrasonics, vol. 36, no. 1-5, pp. 89-96, 1998.

[2] R. Singh and J. S. Khamba, "Ultrasonic machining of titanium and its alloys: a review," Journal of Materials Processing Technology, vol. 173, no. 2, pp. 125-135, 2006.

[3] T. Asami and H. Miura, "Study of ultrasonic machining by longitudinal-torsional vibration for processing brittle materials-observation of machining marks," Physics Procedia, vol. 70, pp. 118-121, 2015.

[4] V. I. Babitsky, V. K. Astashev, and A. Meadows, "Vibration excitation and energy transfer during ultrasonically assisted drilling," Journal of Sound and Vibration, vol. 308, no. 3-5, pp. 805-814, 2007.

[5] L. DeFu, W. L. Cong, Z. J. Pei, and Y. JunTang, "A cutting force model for rotary ultrasonic machining of brittle materials," International Journal of Machine Tools \& Manufacture, vol. 52, pp. 77-84, 2012.

[6] J. Wang, P. Feng, J. Zhang, W. Cai, and H. Shen, "Investigations on the critical feed rate guaranteeing the effectiveness of rotary ultrasonic machining," Ultrasonics, vol. 74, pp. 81-88, 2017.

[7] V. Sharma and P. M. Pandey, "Optimization of machining and vibration parameters for residual stresses minimization in ultrasonic assisted turning of 4340 hardened steel," Ultrasonics, vol. 70, pp. 172-182, 2016.

[8] M. Roopa Rani, K. Prakasan, and R. Rudramoorthy, "Studies on thermo-elastic heating of horns used in ultrasonic plastic welding," Ultrasonics, vol. 55, no. 1, pp. 123-132, 2015.

[9] T. V. da Cunha and C. E. N. Bohórquez, "Ultrasound in arc welding: a review," Ultrasonics, vol. 56, pp. 201-209, 2015.

[10] W. L. Cong, Z. J. Pei, T. W. Deines, A. Srivastava, L. Riley, and C. Treadwell, "Rotary ultrasonic machining of CFRP composites: a study on power consumption," Ultrasonics, vol. 52 , no. 8 , pp. 1030-1037, 2012.

[11] P. Feng, J. Wang, J. Zhang, and J. Zheng, "Drilling induced tearing defects in rotary ultrasonic machining of C/SiC composites," Ceramics International, vol. 43, no. 1, pp. 791-799, 2017.

[12] K. Ding, Y. Fu, H. Su, Y. Chen, X. Yu, and G. Ding, "Experimental studies on drilling tool load and machining quality of $\mathrm{C} / \mathrm{SiC}$ composites in rotary ultrasonic machining," Journal of Materials Processing Technology, vol. 214, no. 12, pp. 2900-2907, 2014.

[13] E. Eisner, "Design of sonic amplitude transformers for large magnification," in Proceedings of the IEEE, vol. 51, pp. 512-512, IEEE.

[14] K. Graff, Wave Motion in Elastic Solids, The Clarendon Press, Oxford, UK, 1975.

[15] D. Sindayihebura, L. Bolle, A. Cornet, and L. Joannes, "Theoretical and experimental study of transducers aimed at lowfrequency ultrasonic atomization of liquids," Journal of the Acoustical Society of America, vol. 103, no. 3, pp. 1442-1448, 1998.

[16] D.-A. Wang, W.-Y. Chuang, K. Hsu, and H.-T. Pham, "Design of a Bézier-profile horn for high displacement amplification," Ultrasonics, vol. 51, no. 29, pp. 148-156, 2011.

[17] G. Amza and D. Drimer, "The design and construction of solid concentrators for ultrasonic energy," Ultrasonics, vol. 14, no. 5, pp. 223-226, 1976.

[18] J. J. Coy and F. S. Tse, "Synthesis of solid elastic horns, transactions of ASME," Journal of Engineering for Industry, vol. 96, no. 2, pp. 627-632, 1974.

[19] X.-P. He and J. Gao, "A review of ultrasonic solid horn design," Technical Acoustics, vol. 25, no. 1, pp. 82-86, 2006.

[20] V. Salmon, "Generalized plane wave horn theory," Journal of the Acoustical Society of America, vol. 17, no. 3, pp. 199-211, 1946.

[21] M. P. Kamat, V. B. Venkayya, and N. S. Khot, "Optimization with frequency constraints-limitations," Journal of Sound and Vibration, vol. 91, no. 1, pp. 147-154, 1983.

[22] J.-C. Hung, Y.-P. Tsai, and C. Hung, "Optimization of ultrasonic plastic welding horns on amplitude uniformity," Applied Mechanics and Materials, vol. 121, no. 126, pp. 278-282, 2012.

[23] T. He, X.-Q. Ye, and Y. Zhao, "Optimization design for ultrasonic horn with large amplitude based on genetic algorithm," Journal of Vibroengineering, vol. 17, no. 3, pp. 1157-1168, 2015.

[24] P. Harkness, A. Mathieson, C. Murray, and M. Lucas, "Optimization of ultrasonic horns for momentum transfer and survivability in high-frequency/low frequency planetary drill tools," in Proceedings of the AIAA SPACE Conference and Exposition 2011, Long Beach, Calif., USA, September 2011.

[25] I. C. Rosca, S. T. Chiriacescu, and N. C. Cretu, "Ultrasonic horns optimization,” Physics Procedia, vol. 3, pp. 1033-1040, 2010.

[26] I.-C. Rosca, M.-I. Pop, and N. Cretu, "Experimental and numerical study on an ultrasonic horn with shape designed with an optimization algorithm," Applied Acoustics, vol. 95, pp. 6069, 2015. 
[27] K.-M. Shu, Y.-G. Li, C.-C. Chan, and J.-B. Kuan, "Optimized design of the horn of ultrasonic roll welding," Advanced Materials Research, vol. 482-484, pp. 2223-2226, 2012.

[28] L. Xu, S. Lin, and W. Hu, "Optimization design of high power ultrasonic circular ring radiator in coupled vibration," Ultrasonics, vol. 51, no. 7, pp. 815-823, 2011.

[29] A. G. Webster, "Acoustical impedance, and the theory of horns and the phonograph," Proceedings of the National Academy of Science of the United States of America, vol. 5, pp. 275-282, 1919.

[30] E. Eisner, "Complete Solutions of the "Webster" Horn Equation," Journal of the Acoustical Society of America, vol. 41, no. 4B, pp. 1126-1146, 1967.

[31] H. Popescu and V. Chiroiu, "Calculul structurilor optimale (Calculation of optimal structures - in romanian)," Editura Academiei Române, Bucuresti (Romanian Academy Printhouse), 1981.

[32] M. J. Turner, "Design of minimum mass structures with specified natural frequencies," AIAA Journal, vol. 5, no. 3, pp. 406-412, 1967.

[33] M. J. Forray, Variational Calculus in Science and Engineering, McGraw Hill Inc., New York, NY, USA, 1968.

[34] V. A. Ilyin and E. G. Poznyak, "Fundamentals of Mathematical Analysis," Mir Publishers, vol. I \& II, 1982.

[35] C. T. Chen, Linear System Theory and Design, Holt, Rinehart and Winston, New York, NY, USA, 1984.

[36] L. M. Silverman, "Realization of linear dynamical systems," IEEE Transactions on Automatic Control, vol. 16, no. 6, pp. 554567, 1971.

[37] B. De Schutter, "Minimal state-space realization in linear system theory: an overview," Journal of Computational and Applied Mathematics, vol. 121, no. 1-2, pp. 331-354, 2000.

[38] D. E. Newland, Mechanical Vibration Analysis and Computation, Longman, Harlow and John Wiley, New York, NY, USA, 1989.

[39] R. Fazlollah, Linear Spaces in Engineering, Ginn \& Company, Waltham, Mass, USA, 1971.

[40] J. He and Z.-F. Fu, Modal Analysis, Butterworth-Heinemann, 2001. 


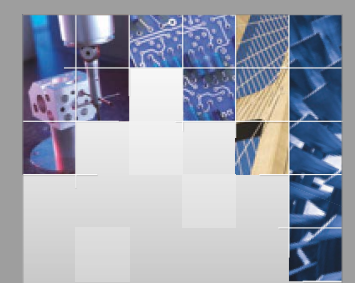

\section{Enfincering}
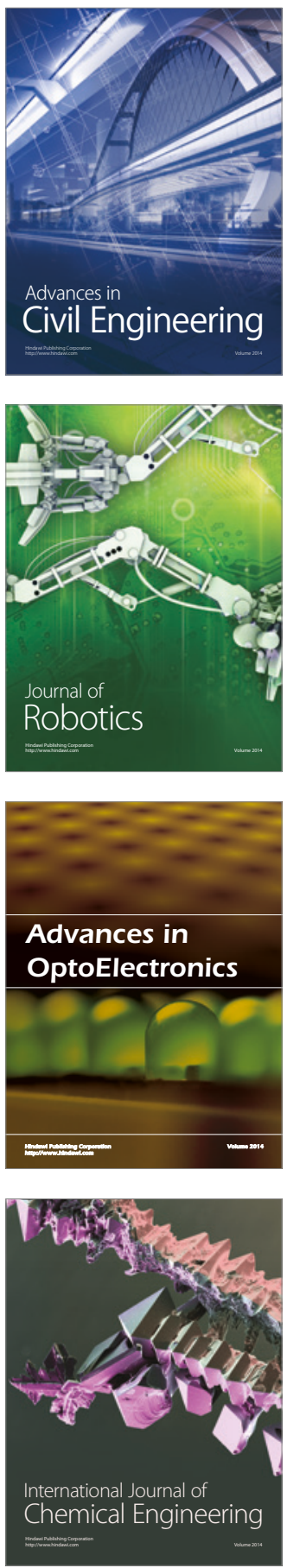

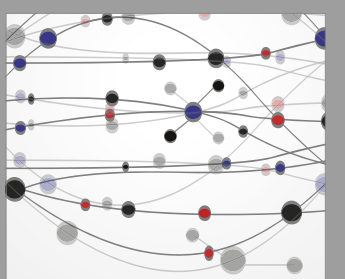

The Scientific World Journal

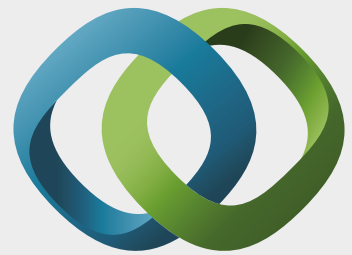

\section{Hindawi}

Submit your manuscripts at

https://www.hindawi.com
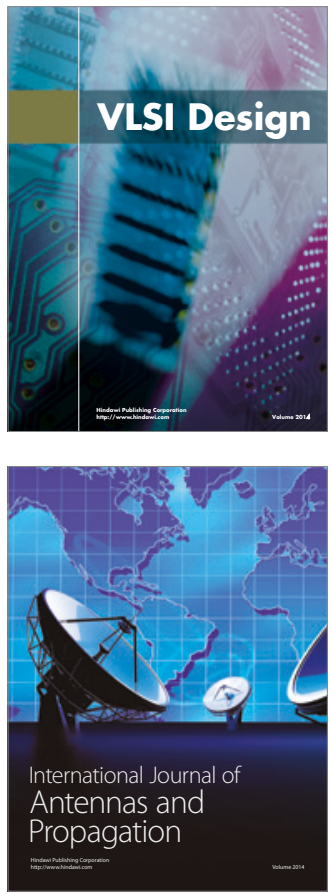

\section{Rotating}

Machinery
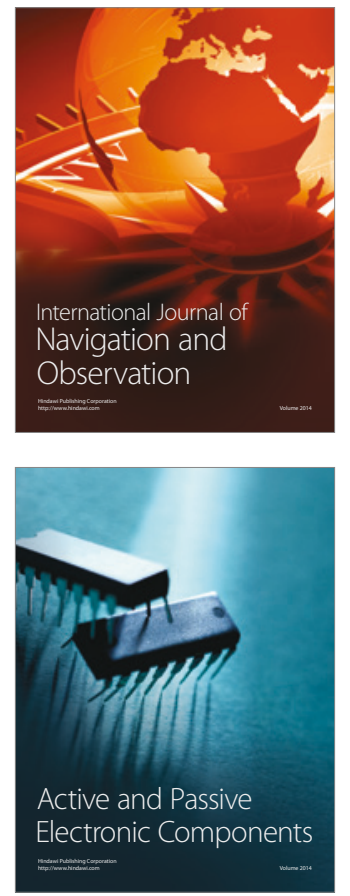
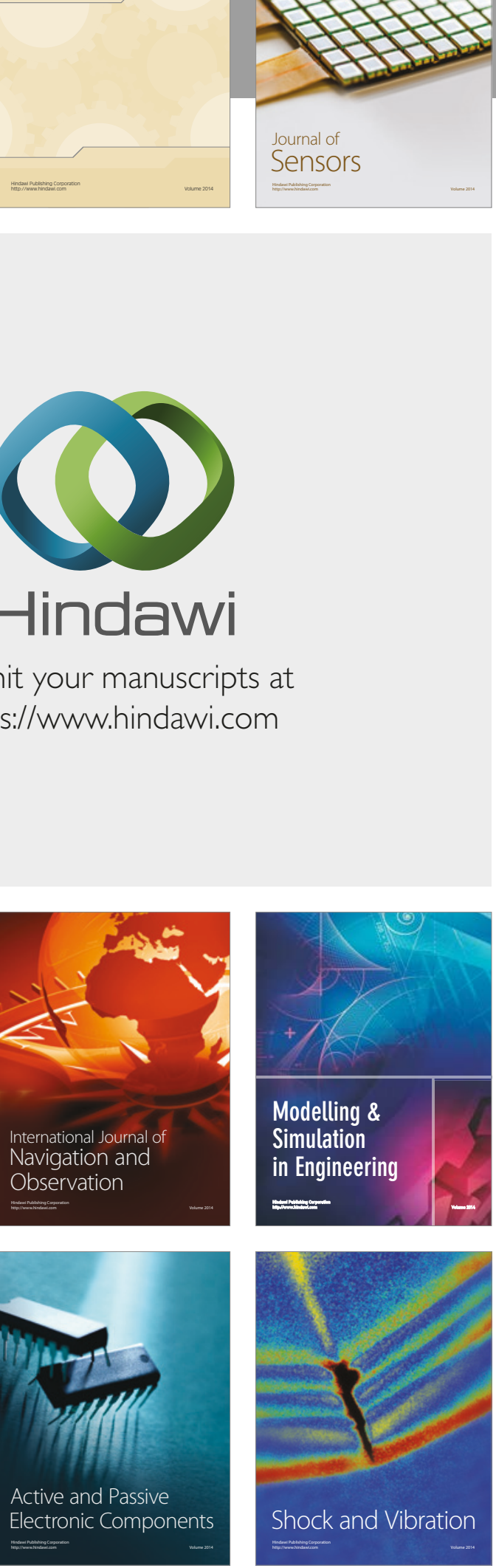
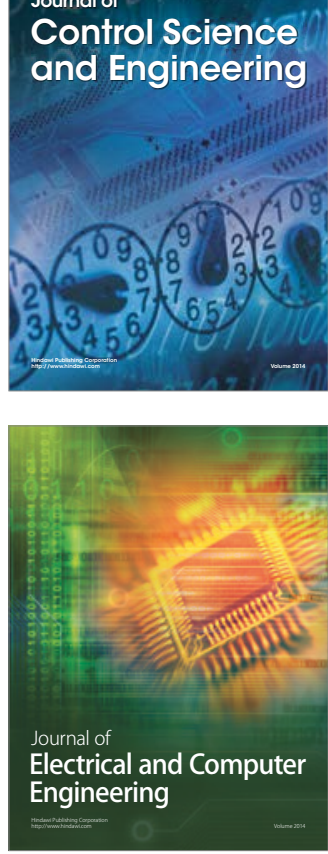

Distributed

Journal of

Control Science

and Engineering
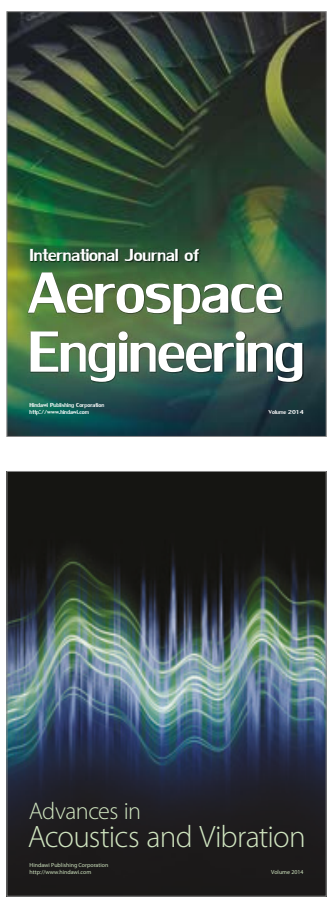

Sensor Networks 